\title{
Submillimetre Astronomy with the James Clerk Maxwell Telescope
}

\author{
Gary Davis \& Antonio Chrysostomou
}

\author{
Joint Astronomy Centre, 660 North A'ohoku Place, Hilo, Hawaii 96720, USA
}

\begin{abstract}
The James Clerk Maxwell Telescope is the world's largest dish designed specifically for use at Submillimetre wavelengths $(0.3-3 \mathrm{~mm})$. It is also, by any objective measure, the world's most productive submillimetre observatory [1]. The telescope, the site, the instrumentation suite and the future scientific programme are presented in this paper.
\end{abstract}

Index Terms - Astronomy, submillimetre wave antennas, submillimetre wave receivers.

\section{INTRODUCTION}

The James Clerk Maxwell Telescope (JCMT) is the world's premier ground-based facility for astronomical observations at submillimetre wavelengths. This status is afforded by three essential ingredients: the size and quality of the telescope, its location, and its aggressive programme of instrumentation. Each of these is discussed in the following paragraphs.

The JCMT is the largest single-dish telescope in the world designed specifically to operate in the submillimetre region of the spectrum (wavelength range $300 \mu \mathrm{m}$ to $3 \mathrm{~mm}$ ). The primary reflector of the JCMT has a diameter of $15 \mathrm{~m}$ and is made up of 276 individual lightweight panels. Each panel consists of a thin aluminium skin bonded to an aluminium honeycomb and is attached to at three points to the backing structure of the antenna. The backing structure is designed to maintain a parabolic figure as gravity distorts the antenna as it tips to different elevations. The alignment of individual panels can be adjusted by means of actuators at the mounting points. The sub-reflector, or secondary mirror, can be adjusted in three axes to compensate for changes in focus as well as changes in the figure of the primary. In addition, the secondary can be tilted or chopped in two axes in order to perform sky background cancellation.

In order to protect the telescope from the environment, the entire structure is enclosed within a building which co-rotates with the antenna and provides a safe and comfortable working environment. During observing periods the roof and doors are opened and a membrane (the world's largest piece of GoreTex) is normally deployed in front of the antenna. The membrane is transparent at millimetre and submillimetre wavelengths. In addition to providing protection from the wind, the membrane performs the useful function of reflecting the visible and near-infrared radiation, providing some protection from solar heat which could damage the antenna and the thermal balance of the telescope, thereby allowing daytime astronomical observations to occur including direct observations of the Sun itself.
The facility is situated at the summit of Mauna Kea, on the island of Hawaii, at an altitude of $4092 \mathrm{~m}$. This is one of the best sites in the world for submillimetre astronomy, and certainly the very best in the northern hemisphere. The strongest constraint on ground-based observing at submillimetre wavelengths is the absorption of astronomical signals by water vapour in the Earth's atmosphere. The choice of site is vitally important in order to minimise this effect and to maximise the scientific productivity of the facility. Since the summit of Mauna Kea is the highest point in the Pacific Ocean, the telescope is physically above $97 \%$ of the water vapour in the atmosphere. Its distance from sources of industrial and city light pollution as well as its exceptional weather characteristics makes Mauna Kea one of the premier observing sites in the world.

Instruments for the telescope can be located within bays of the Cassegrain cabin or on one of the two Nasmyth platforms located at the ends of the elevation bearings. Two categories of instrument are provided for users of the facility. Heterodyne receivers are available to measure line emission from specific molecules, revealing physical and chemical information about the source being observed (composition, temperature, velocity). Imaging photometric instruments measure thermal emission from cold dust at wavelengths of 450 and $850 \mu \mathrm{m}$, enabling investigation of a wide variety of astrophysical phenomena. In addition, polarimeters are available for both categories of instrument, which makes possible the study of magnetic fields in space.

The JCMT is a joint project of three countries: the United Kingdom (55\%), Canada (25\%) and the Netherlands (20\%). Each country contributes, in the stated proportion, to the operating budget of the facility and to the development of the instrumentation. Responsibility for the operation and development of the telescope rests with the Director JCMT. The administrative base for the facility is the Joint Astronomy Centre (JAC), an establishment of the UK Science and Technology Facilities Council (STFC), located at sea level in Hilo, Hawaii.

\section{THIRD-GENERATION INSTRUMENT SUITE}

The JCMT is currently in the midst of an ambitious upgrade programme in which the entire instrument suite is being replaced. This upgrade represents an investment of roughly $\$ 35 \mathrm{M}$. 


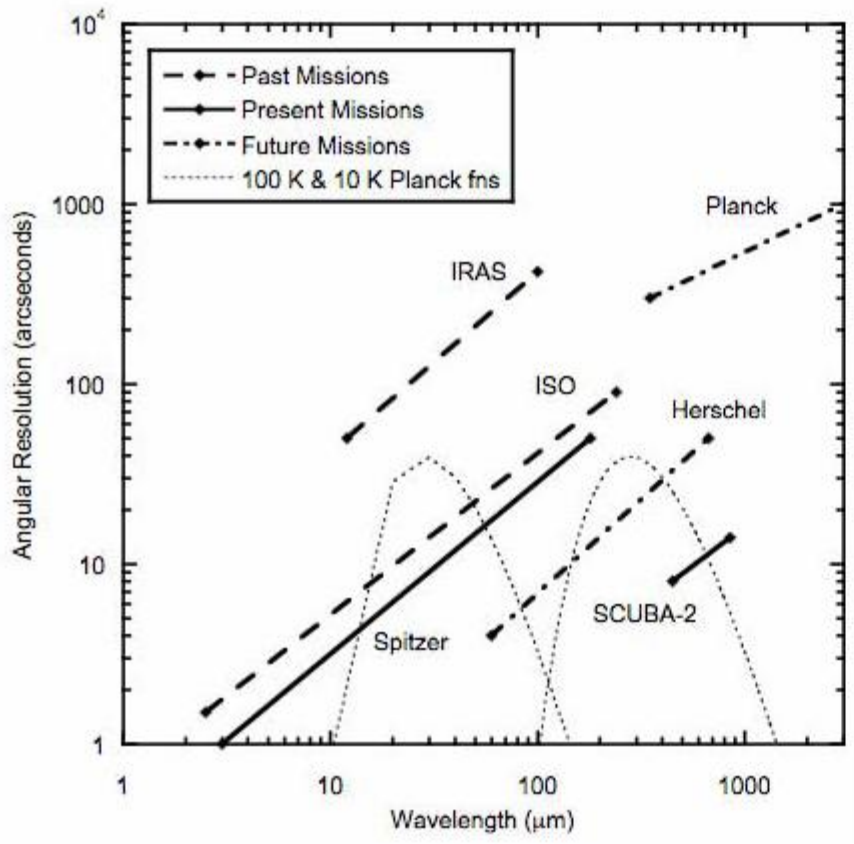

Fig. 1. The angular resolutions of past, present and future missions (funded). SCUBA-2 is the only ground-based facility shown here. The dotted lines show Planck functions for dust emitting at $100 \mathrm{~K}$ and $10 \mathrm{~K}$. With its sensitivity and mapping speed, SCUBA-2 will efficiently survey the sky for the coldest objects in our Galaxy and the universe at the highest spatial resolution.

The flagship instrument of this development programme is a new continuum camera called SCUBA-2, the successor to SCUBA which was retired in 2005. SCUBA was the first imaging camera in the world designed to operate at submillimetre wavelengths, and as such it was responsible for many new discoveries spanning a wide range of astrophysical phenomena. One example is the imaging of debris disks around nearby stars, from which the presence of planetary systems can be inferred. Another is the discovery, shortly after SCUBA was commissioned, of a large population of massive, dust-enshrouded galaxies in the early universe, a class of object now commonly referred to as "SCUBA galaxies". SCUBA was one of the most successful and productive ground-based astronomical instruments of modern times: it accounted, over its 8-year lifetime, for roughly $75 \%$ of all publications based on JCMT data, and was at one stage ranked second only to the Hubble Space Telescope in terms of its impact on astronomy [1].

SCUBA-2 will build on this legacy by providing enhanced sensitivity over a much larger field of view. The sensitivity improvement is obtained by using transition-edge superconductors as the sensing elements. These devices make use of the strong dependence of electrical resistance to temperature at the superconducting transition point. This transition occurs at a temperature of $100 \mathrm{mK}$, which will be generated by a dilution refrigerator. The field of view will exceed 50 square arc minutes and is achieved by a novel and complex optical design which aims to use as much of the telescope's unvignetted field of view as possible. These innovations, in combination with improved sampling and sky scanning techniques, will bring CCD-like imaging to the submillimetre for the first time. We currently estimate that SCUBA-2 will be able to map large regions of the submillimetre sky up to 1000 times faster than SCUBA. Taking a wider perspective, Figure 1 shows how the angular resolution of past, present and future (and funded) infrared survey missions compare. At the longest infrared wavelengths where cold dust emits brightest, SCUBA-2 is in a unique position to make the most significant advances.

In parallel with this, the heterodyne capability of the telescope is also being upgraded. We recently took delivery of HARP, the world's first array receiver operating in the 345 $\mathrm{GHz}$ atmospheric window. With its high spectral resolution and $4 \times 4$ array of receptors, based on SIS superconducting tunnel junctions, HARP will provide a new spectral imaging capability. By providing spectral information in addition to morphology, HARP will probe the physical, dynamical and chemical conditions in molecular clouds and the interstellar medium in our own and nearby galaxies. HARP will be used in conjunction with ACSIS, a new multi-channel backend correlator system. The ACSIS/HARP combination will be highly complementary to SCUBA-2, enabling detailed spectroscopic follow up of sources detected in large-scale photometric maps.

Finally, three new "ancillary" instruments are being developed for use in conjunction with HARP and SCUBA-2: ROVER, a spectral line polarimeter; POL-2, a continuum polarimeter; and FTS-2, a low-resolution Fourier transform spectrometer. These instruments will supplement and extend the capabilities of HARP and SCUBA-2 by providing additional physical information about sources under study. The entire suite of instrumentation is both comprehensive and versatile, and is unmatched in scope by any other submillimetre observatory.

\section{THE JCMT LEGACY SURVEY PROGRAMME}

The third generation instruments described above have all been optimized for mapping large regions of the submillimetre sky. Despite the great success of SCUBA, for example, less than 25 square degrees of the sky have been observed at submillimetre wavelengths (see Figure 2). The time has come to build on the discoveries made with SCUBA and other instruments by extending our observations to larger, statistically significant samples. To give just one example, roughly 100 SCUBA galaxies have been detected and characterized; with SCUBA-2 we expect to detect thousands of such objects, sufficient to measure galaxy clustering properties and hence infer the evolution of large-scale structure in the universe.

In recognition of these unprecedented capabilities, and in order to make best use of the large amounts of telescope time 


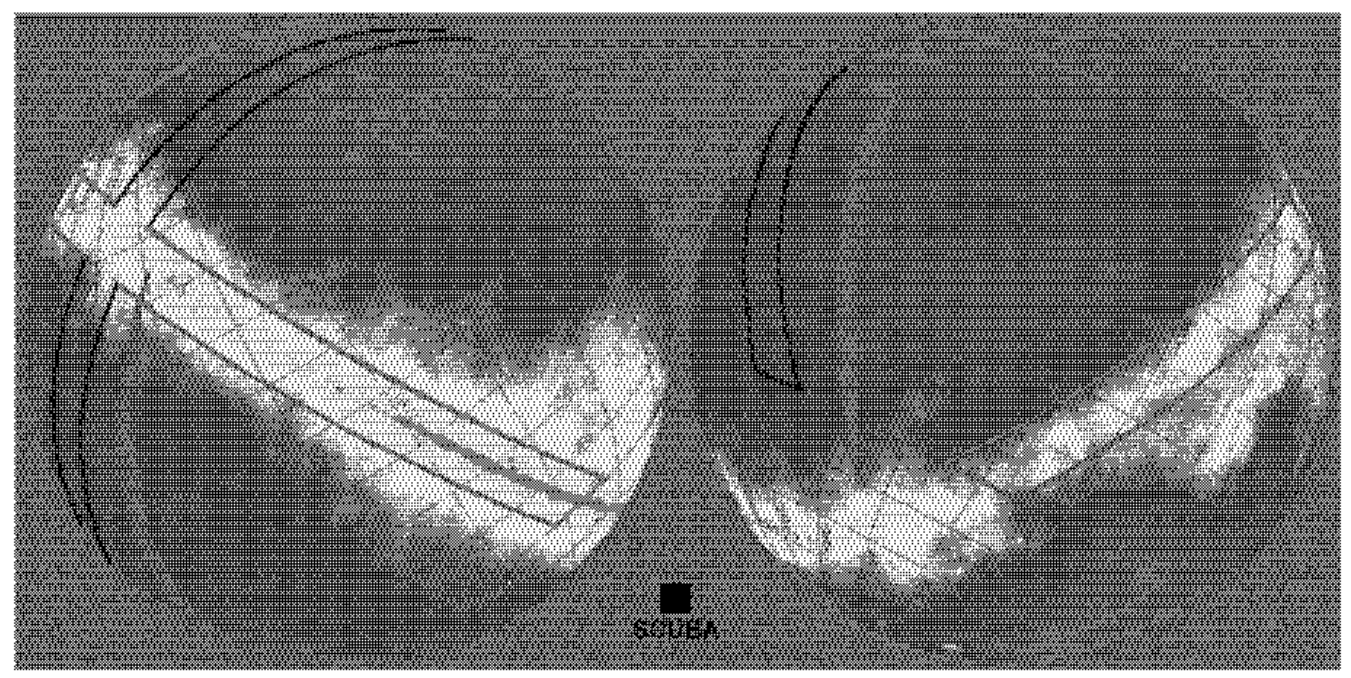

Fig. 2. The image shows a representation of the infrared sky as mapped by the IRAS satellite at $100 \mu \mathrm{m}$. The regions outlined by the red and black strips will be mapped as part of the SCUBA-2 'All sky' Survey, within two years. For comparison, the black square corresponds to the total area of the sky mapped by SCUBA in its seven year lifetime. Figure courtesy of Mark Thompson.

which would be required, the astronomical communities in the three partner countries have jointly developed the JCMT Legacy Survey programme consisting of seven separate projects. While we provide quick descriptions of each of the seven surveys here, more detailed summaries are available at http://www. jach. hawaii.edu/jcmt/surveys.

\section{A. Spectral Legacy Survey}

This is a project to use HARP to study the molecular inventory and physical structure of a sample of objects spanning different evolutionary stages and different physical environments in molecular clouds. This project will aid our understanding of the chemical evolution of that occurs during the star formation process.

\section{B. Nearby Galaxies Survey}

This project will use a combination of SCUBA-2 and HARP to study the interstellar medium and its interplay with star formation in a sample of nearby galaxies.

\section{Debris Disk Survey}

This project will use SCUBA-2 to conduct the first unbiased survey of debris disks around 500 nearby main-sequence stars, providing robust statistics on the incidence of such disks.

\section{Gould's Belt Survey}

This comprehensive project will map most of the star forming regions within $500 \mathrm{pc}$ of the Earth using SCUBA-2. The most significant regions from this survey will also be observed with HARP and POL-2 in order to better understand the interplay of gas kinematics and the magnetic field in molecular clouds.

\section{E. JCMT Galactic Plane Survey}

This project will address key questions in massive star formation by mapping two-thirds of the Galactic Plane in the submillimetre continuum.

\section{F. Cosmology Survey}

This project will build on the success of the SCUBA cosmology surveys by making the deepest submillimetre maps at $450 \mu \mathrm{m}$ and $850 \mu \mathrm{m}$. It is designed around a simple 2-tier survey, located in a set of well-defined fields with appropriately low far-infrared background, with extensive multi-frequency supporting data. This survey programme will revolutionize our understanding of sub-mm galaxies, and galaxy formation in general and provide cosmologists with a uniquely powerful resource for the exploitation of the new and growing range of public survey datasets.

\section{G. SCUBA-2 'All-Sky' Survey}

This is an ambitious project with ultimate aims to map the entire sky visible from the JCMT (18,000 square degrees) using SCUBA-2. It will do so with minimum impact on normal JCMT operations by utilizing the poorest weather conditions on Mauna Kea, thereby providing a comprehensive data set of value to both cosmology and Galactic studies.

\section{CONCLUSION}

The extraordinary quality and scope of the JCMT Legacy Survey is self-evident: it is of the highest scientific calibre. The surveys will revolutionise our understanding of planet formation processes; they will address questions fundamental to star formation studies, and obtain a molecular inventory of objects spanning a range of evolutionary stages and physical environments; they will produce the first large sample of local 
galaxies observed with good spatial resolution at submillimetre wavelengths; the entire sky visible to JCMT will be mapped, including deep scans of the accessible Galactic Plane, placing massive star formation research onto a firm statistical basis for the first time. Last, but not least, the cosmology programme will transform our understanding of galaxy formation, yielding a survey of enormous and lasting legacy value. The knowledge gained from the JCMT Legacy Survey will have far-reaching benefits for the whole of astrophysics.

\section{REFERENCES}

[1] V. Trimble, P. Zaich, T. Bosler, "Productivity and Impact of Optical Telescopes," PASP, 117, 111-118, 2005. 